\title{
Desgaste psíquico (burnout) en profesores de educación primaria de Lima metropolitana
}

Manuel Fernández

Universidad de Lima

Lima, Perú

El presente estudio explora el síndrome de desgaste psíquico (burnout) en una muestra de 264 profesores de educación primaria de Lima metropolitana. Se analizan las tres dimensiones que comprende el síndrome: agotamiento emocional, despersonalización y falta de realización personal. Los resultados indican que un $43 \%$ de profesores alcanzan niveles altos en el síndrome del desgaste psíquico. Se discuten las diferencias encontradas en cada una de las dimensiones del síndrome en relación con una serie de variables sociodemográficas en las que se encuentra inmerso el maestro.

desgaste psíquico / maestro / escuela primaria

\section{Psychological burnout syndrome in elementary school teachers in metropolitan Lima}

This research studies the psychological burnout syndrome on a sample of 264 elementary school teachers. Three components of the syndrome are analyzed: emotional exhaustion, depersonalization and lack of self-fulfillment. Results indicate that $43 \%$ of teachers are prone to develop the syndrome in a short lapse of time. The components of the syndrome are discussed and related to sociodemographic variables in which the teachers are immersed.

psychological burnout / teacher / elementary school

Dirección del autor: mfernand@correo.ulima.edu.pe 
La actividad del docente comprende diversidad de tareas, como la preparación y dictado de clases; coordinar con otros profesores y autoridades del centro educativo las actividades académicas y extraacadémicas; evaluar a los estudiantes, atender las consultas de los padres de familia, preparar reportes periódicos de su labor, cumplir con las normas y procedimientos que emanan de los organismos educativos, así como participar en los programas de capacitación o actualización docente.

El profesor debe enfrentar una serie de dificultades económicas y sociales que, en realidad, afectan intensa y permanentemente a una amplia mayoría de los maestros de países subdesarrollados como el nuestro, que le impiden disponer del tiempo y la tranquilidad necesarios para preparar sus clases y exámenes, así como para desarrollar eficientemente las demás actividades que le competen.

Dada la compleja situación en la que se encuentra el maestro, en la cual las demandas laborales y personales escapan a su real capacidad, las posibilidades de padecer de estrés o llegar al agotamiento emocional o psíquico resultan bastante elevadas.

El concepto de desgaste psíquico o "quemarse por el trabajo" comenzó a estudiarse en Estados Unidos por los años setenta, para tratar de explicar el proceso de deterioro en los cuidados y atención profesional a los usuarios de las organizaciones de servicios.
Desde la perspectiva psicosocial (Maslach \& Jackson, 1981), el desgaste psíquico se refiere a un síndrome de agotamiento emocional, despersonalización y falta de realización personal en el trabajo que puede desarrollarse en personas cuyo objeto de trabajo son personas en cualquier tipo de actividad de servicio.

El estudio de esta variable en los profesores de educación primaria puede resultar relevante, dadas las diversas demandas organizacionales y personales a las que tienen que enfrentarse por la naturaleza del trabajo que desempeñan, así como por la relación que establece con los estudiantes.

\section{Desgaste psíquico (BURNout)}

El término "desgaste psíquico" (burnout) fue empleado por primera vez por Freudenberger (1974) para describir un estado particular de agotamiento físico y psicológico en los trabajadores de la salud. El burnout se refiere al proceso de deterioro en los cuidados y atención profesional a los usuarios de las organizaciones de servicios (Gil \& Peiró, 1997). Los síntomas aparecen en profesionales que brindan atención social, educativa o sanitaria a otras personas en términos de una relación de ayuda, consejo o enseñanza (Salgado $e t$ al., 1997).

Edelwich \& Brodsky (1980) han propuesto un modelo progresivo del burnout con cuatro etapas. En la primera, el idealismo y el entusiasmo llevan al 
trabajador a plantearse expectativas irreales con respecto a lo que puede conseguir con su labor. La segunda, caracterizada por el estancamiento, tiene como elemento fundamental una paralización al constatar la irrealidad de las expectativas, con lo cual el idealismo y el entusiasmo iniciales desaparecen. La tercera etapa, central en el burnout, es de frustración. La cuarta y última es de apatía y de un sentimiento de vacío que se refleja en la calidad y en la iniciativa en el trabajo.

El burnout puede entenderse como una respuesta al estrés laboral crónico, integrado por actitudes y sentimientos negativos hacia las personas con las que se trabaja (actitudes de despersonalización), hacia el propio rol profesional (falta de realización personal en el trabajo) y por la vivencia de encontrarse emocionalmente agotado. Esta respuesta ocurre en los profesionales que prestan servicios (médicos, maestros, funcionarios de prisiones, policías, trabajadores sociales, etc.) en contacto directo con los usuarios de estos servicios (pacientes, alumnos, presos, indigentes, etc.). Sin embargo, este concepto también ha sido descrito en diferentes tipos de profesionales, como directivos $\mathrm{y}$ mandos intermedios, entrenadores, deportistas e, incluso, fuera del ámbito laboral (voluntarios, amas de casa).

Maslach \& Jackson (1981) trataron de orientar su investigación hacia la identificación de las condiciones ambientales de trabajo que conducen al burnout. Se interesaron en investigar la forma en que el estrés relacionado con el rol laboral puede llevar a una persona a experimentar fatiga mental, a dar un trato "mecanizado" a los clientes y autopercibir disminuida su habilidad para lograr éxito en el trabajo.

Estos autores definen el burnout como un síndrome de agotamiento emocional, despersonalización y falta de realización personal en el trabajo. Este síndrome es causado por estresores organizacionales (Vandenberghe \& Huberman, 1999), y se puede desarrollar en personas cuyo objeto de trabajo son también personas. A diferencia de Freudenberger, Maslach \& Jackson consideran que el burnout provoca en el trabajador una tendencia a tratar a sus clientes de una manera desprendida y deshumanizada.

En un principio, el síndrome de burnout se entendía como un estado; sin embargo, actualmente se entiende como proceso en el que se incluyen las tres dimensiones mencionadas.

El agotamiento emocional está referido a una disminución o deficiencia en los recursos emocionales propios; los trabajadores sienten que ya no pueden dar más de sí a nivel afectivo. Esta carencia de recursos emocionales propios se debe al contacto diario y sostenido con personas a las que se atiende como objeto de trabajo (pacientes, alumnos, etc.). La despersonalización se define como una respuesta excesivamente negativa, insensible y despreocupada ha- 
cia las otras personas caracterizada por actitudes de cinismo hacia ellas. Debido a un endurecimiento afectivo, las personas son vistas de forma deshumanizada, culpándolas de sus problemas (por ejemplo, el paciente se merece la enfermedad, el preso se merece la condena, etc.). La falta de realización personal se relaciona con el deterioro de los propios sentimientos de competencia y éxito en la realización del propio trabajo. Así, los profesionales tienden a evaluarse de manera negativa, afectando la realización de su trabajo y la relación con las personas que son objeto de éste.

Para explicar la etiología del burnout como respuesta al estrés laboral, se han desarrollado una serie de teorías y modelos, algunos de los cuales pasamos a revisar:

- Modelo desde la teoría sociocognitiva del yo

Este modelo se basa en los planteamientos de Bandura (1989), y sostiene que existen procesos de asociación por los cuales las representaciones simbólicas se traducen en acciones. De acuerdo con esto, los modelos para explicar la etiología del síndrome del burnout señalan que:

- Las cogniciones de las personas influyen en lo que perciben y hacen, y se modifican por los efectos de las acciones y por la acumulación de las consecuencias observadas en los demás.
- El grado de seguridad de la persona en sus propias capacidades determinará el empeño que la persona pondrá para conseguir sus objetivos y la facilidad o dificultad en conseguirlos, y también determinará ciertas reacciones emocionales como la depresión o el estrés que acompañan la acción.

- Modelo de competencia social Harrison (1983, en Gil \& Peiró, 1997) sostiene que la competencia y la eficacia percibidas son los principales factores asociados a este síndrome. Señala que las personas que trabajan en servicios de ayuda están muy motivadas para ayudar a otros $\mathrm{y}$ tienen un sentimiento elevado de altruismo. En el entorno laboral, las personas se encuentran con factores de ayuda que facilitan la actividad del sujeto o factores barrera que la dificultan. En un principio, la motivación para ayudar predeterminará la eficacia del sujeto en la consecución de los objetivos laborales. Si en el entorno existen factores de ayuda esta efectividad crecerá y aumentarán los sentimientos de competencia social del sujeto. De este modo, los sentimientos de eficacia aumentarán, influyendo positivamente en la motivación para ayudar. Sin embargo, cuando las personas encuentran factores barrera, en el entorno ocurrirá una disminución de los sentimientos de eficacia, ya que 
el sujeto no logra conseguir sus objetivos, afectando de manera negativa la esperanza de conseguirlos. Si esta situación se mantiene en el tiempo, surge el síndrome de burnout, el cual, a su vez, facilita el desarrollo de los factores barrera, disminuye los sentimientos de eficacia percibida y la motivación para ayudar.

Pines (1993) plantea que sólo las personas que intentan darle un sentido existencial a su trabajo llegarán a sufrir de burnout. El sentido existencial se refiere a aquellos casos en que los profesionales justifican su existencia por la labor de carácter humanitario y de ayuda que desarrollan en su trabajo, así, esta justificación hace que la vida tenga sentido para la persona, ya que las cosas que hace son útiles e importantes. Pines (citado por Gil \& Peiró, 1997) señala que el burnout es resultado de un proceso de desilusión, una falla en la búsqueda de sentido para la vida en personas muy motivadas hacia su trabajo. Una persona que no esté inicialmente muy motivada puede experimentar estrés, fatiga o depresión, pero no el síndrome de burnout.

Para Cherniss es posible establecer una relación causal entre la incapacidad del sujeto para desarrollar sentimientos de competencia o éxito personal y el síndrome de burnout. Para esto incorpora el concepto de autoeficacia percibida de Bandura, defini- da como las creencias que tienen las personas sobre sus capacidades para controlar las situaciones que las afectan. Al entender el papel de la autoeficacia en la etiología del síndrome, se puede explicar por qué algunas características del entorno laboral conducen al síndrome.

El logro de los objetivos personales aumenta los sentimientos de autoeficacia. Por el contrario, el fallo en alcanzar los objetivos conlleva sentimientos de fracaso, baja autoeficacia y, por lo tanto, se puede desarrollar el síndrome de burnout. Cherniss afirma que existe una vinculación entre sentimientos de autoeficacia, compromiso y motivación, y entre aquéllos y el estrés. Los sujetos con fuertes sentimientos de autoeficacia percibida experimentan menos estrés en situaciones amenazantes y las situaciones son menos estresantes cuando las personas creen que pueden afrontarlas exitosamente. Al considerarse el síndrome de burnout como una respuesta al estrés, la relación entre la autoeficacia y estrés sugiere una relación con el síndrome de burnout.

- Modelo de Thompson, Page \& Cooper (1993)

Los autores señalan que la etiología del síndrome está relacionada con cuatro variables:

- las discrepancias entre las demandas de la tarea y los recursos del sujeto, 
- el nivel de autoconciencia de la persona,

- las expectativas de éxito de la persona, $\mathrm{y}$

- los sentimientos de autoconfianza de la persona.

La autoconciencia se considera fundamental en la etiología del síndrome y es entendida como la capacidad del sujeto de autorregular sus niveles de estrés percibido durante el desarrollo de una tarea dirigida a la consecución de un objetivo. Thompson et al. (1993) proponen que el reconocimiento de las discrepancias percibidas entre demandas y recursos puede intensificar el nivel de autoconciencia en algunos sujetos, lo que afectará negativamente su estado anímico, causando también una falta de confianza percibida para resolver esas discrepancias y bajos sentimientos de realización personal en el trabajo.

Cuando la consecución de los objetivos se ve continuamente frustrada, las personas con un alto rasgo de autoconciencia y pesimismo tienden a resolver las dificultades retirándose de manera mental o conductual de la situación problemática. La despersonalización es considerada como un patrón de retiro conductual, mientras que el agotamiento emocional es entendido como un retiro psicológico. Rasgos intensos de autoconciencia y pesimismo en los profesionales potencian el retiro del esfuerzo que deberían invertir para resolver las discrepancias. Los autores señalan que altos niveles de autoconciencia aumentan la experiencia de desilusión, frustración y pérdida.

- Modelo de Golembyewski, Munzenrider \& Carter (1983)

Estos autores definen el síndrome de burnout como un proceso en el que los profesionales pierden el compromiso inicial que tenían con su trabajo como una forma de respuesta al estrés laboral y a la tensión que les genera.

El estrés laboral en relación con el burnout se genera por situaciones de sobrecarga laboral (mucha estimulación y reto) y por pobreza de rol (poca estimulación y reto). En ambos casos las personas sienten que pierden autonomía y control, afectando su autoimagen, sintiéndose irritables y fatigados. En la segunda fase las personas desarrollan estrategias de afrontamiento. Estas estrategias incluyen un distanciamiento del profesional de la situación laboral estresante. Este distanciamiento puede ser de carácter constructivo o contraproductivo. Es constructivo cuando el profesional atiende al usuario sin implicarse en el problema, pero sin eliminar la empatía. Es contraproductivo cuando el profesional muestra indiferencia emocional, cinismo, rigidez en el trato o ignora a la persona. Cuan- 
do esto sucede, se habla del síndrome de burnout, pues este tipo de distanciamiento produce actitudes de despersonalización.

Cuando las condiciones de sobrecarga o pobreza de rol permanecen en el tiempo se desarrollan las actitudes de despersonalización, posteriormente la persona desarrollará una experiencia de baja realización personal en el trabajo y, finalmente, agotamiento emocional. Estos autores sostienen que el síndrome de burnout se puede desarrollar en todo tipo de profesionales y no sólo en aquellos que trabajan en contacto directo con las personas.

- Modelo de Cox, Kuk \& Leiter (1993, citado por Gil \& Peiró, 1997)

Este modelo asume una perspectiva transaccional del estrés laboral. Considera que el burnout es un episodio dentro del proceso de estrés laboral que se da específicamente entre profesionales de servicios humanos y se desarrolla cuando las estrategias de afrontamiento empleadas por el sujeto no resultan eficaces para manejar el estrés laboral y sus efectos. Para estos autores la experiencia de agotamiento emocional es una dimensión central del síndrome. La despersonalización es considerada como una estrategia de afrontamiento, que surge frente al agotamiento emocional. Los sentimientos de baja realización personal en el trabajo son el resultado de la evaluación cognitiva del sujeto sobre su experiencia de estrés y están relacionados con el significado del trabajo y con las expectativas profesionales frustradas.

- Modelo de Winnubst (1993)

Winnubst (1993, citado por Gil \& Peiró, 1997) sigue los planteamientos de Pines y Aronson (1988, citado por Gil \& Peiró, 1997), y entiende el síndrome de burnout como un sentimiento de agotamiento físico, emocional y mental, como resultado de la tensión emocional crónica derivada del estrés laboral. No acepta el concepto de tres dimensiones elaborado por Maslach y Jackson (1981). El autor considera que el síndrome del burnout afecta a todo tipo de profesionales y no sólo a los que brindan servicio de ayuda.

Winnubst se centra en las relaciones entre la estructura de la organización, la cultura y el clima organizacional y el apoyo social en el trabajo como variables antecedentes del síndrome del burnout. La estructura organizacional, la cultura y el apoyo social se rigen por criterios éticos derivados de los valores sociales y culturales de la organización. Estos criterios éticos hacen posible predecir la medida en que las organizaciones inducirán en sus miembros tensión y el síndrome de burnout.

- Modelo estructural considerando antecedentes de nivel personal, interpersonal y organizacional 
Para Gil y Peiró (1997), una única perspectiva teórica no parece dar una explicación completa sobre la etiología del síndrome, por lo que trata de integrar las variables recogidas en las tres perspectivas analizadas.

Ellos adoptan un enfoque transaccional, logrando integrar en un modelo el papel de las variables organizacionales, personales y las estrategias de afrontamiento en el proceso del estrés laboral, y plantean el síndrome de burnout como una respuesta al estrés laboral percibido que surge tras un proceso de reevaluación cognitiva, cuando las estrategias de afrontamiento empleadas por los profesionales no son eficaces para reducir ese estrés laboral percibido (Gil \& Peiró, 1997, 44). Esta respuesta supone una variable mediadora entre el estrés percibido y sus consecuencias.

A continuación se presentarán diferentes variables estudiadas por los investigadores, las cuales muestran que el burnout es función de diferentes estresores que actúan tanto a nivel personal como organizacional.

En base a los resultados obtenidos en los diferentes estudios, se observa que existen diferencias significativas en función del género en las actitudes de despersonalización. Los hombres obtienen puntajes más altos en despersonalización que las mujeres, tanto en el nivel de primaria como de secundaria
(Anderson \& Iwanicki, 1984; Burke \& Greenglass, 1989; Byrne, 1991; Greenglass \& Burke, 1990; Ogus et al., 1990; Russel et al., 1987; Schwab \& Iwanicki, 1982; Schwab et al., 1986, citados por Vandenberghe \& Huberman, 1999).

Otros investigadores (Schwab \& Iwanicki, 1982; Russell, 1987, citados por Vandenberghe \& Huberman, 1999) no encontraron diferencias significativas entre los profesores de primaria y secundaria. Los diversos estudios con relación al constructo global de burnout, producen también resultados contradictorios con relación a las diferencias de género (Gessaroli, 1989; Beer \& Beer, 1992; Capel, 1992, citados por Vandenberghe \& Huberman, 1999) y al parecer los resultados se verían influidos por las características específicas de cada estudio (muestra, instrumento, país, cultura, etc.) Se observa que en algunos trabajos el síndrome de burnout es mayor en las mujeres que en los hombres; en otros estudios se obtienen resultados inversos, mientras que algunos no obtienen diferencias significativas en función del género. Así, los hallazgos con esta variable son inconsistentes. $L a$ edad ha demostrado ser una variable que permite establecer diferencias con referencia al agotamiento emocional. Algunos autores encuentran que los profesores jóvenes presentan mayores niveles de agotamiento emocional (Anderson \& Iwanicki, 1984; Maslach \& Jackson, 1981; Russell et al., 1987; Schwab 
et al., 1986, citados por Vandenberghe \& Huberman, 1999).

Sin embargo, otros hallazgos señalan que esto se cumple sólo entre profesores universitarios (Byrne, 1991, citado por Vandenberghe \& Huberman, 1999). Los otros dos componentes presentan hallazgos menos consistentes. Los jóvenes profesionales dedicados al servicio social presentaron baja realización personal (Maslach \& Jackson, 1981, citados por Vandenberghe \& Huberman, 1999). Byrne (1991, citado por Vandenberghe \& Huberman, 1999) encuentra resultados similares tanto para profesores de educación primaria como universitaria.

Asimismo, se encuentra que los jóvenes tienen mayores niveles de despersonalización (Maslach \& Jackson, 1981; Pierce \& Molloy, 1990 citados por Vandenberghe \& Huberman, 1999); sin embargo, Byrne no encuentra diferencias significativas en esta dimensión del burnout. Otros autores tampoco encuentran diferencias significativas con relación a la edad y estas dimensiones (Anderson \& Iwanicki, 1984; Schwab \& Iwanicki, 1982 citados por Vandenberghe \& Huberman, 1999). Si bien los resultados en función de la variable edad no son concluyentes, se observa un número de estudios que indican que a mayor edad los sujetos expresan menos sentimientos de burnout.

En algunos estudios en los que se trabajaron con edades estratificadas se encuentra que el índice de burnout es ba- jo entre los 20-25 años, es alto desde los 25 hasta los 40 años, y es muy pequeño a partir de los 40 años, aproximadamente (Farber, 1984).

Los resultados en relación con los años de experiencia son contradictorios. Se ha encontrado que los profesores con más de 20 años de experiencia presentan mayores niveles de estrés que las personas con menos experiencia (Borg \& Falzon, 1989, citados por Vandenberghe \& Huberman, 1999). Sin embargo, un estudio en profesores de primaria y secundaria muestra que los años de experiencia no contribuyen de manera significativa en el estrés laboral (Malik et al., 1991, citado por Vandenberghe \& Huberman, 1999). Anderson e Iwanicki (1984, citados por Vandenberghe \& Huberman, 1999) reportaron menores niveles en la percepción de logros personales en los profesores entre 13 y 24 años de experiencia. No se encontraron hallazgos significativos para la despersonalización ni para el agotamiento emocional (Schwab \& Iwanicki, 1982, citados por Vandenberghe \& Huberman, 1999).

Schwab e Iwanicki (1982, citados por Vandenberghe \& Huberman, 1999) no encontraron efectos significativos del estado marital entre los profesores de primaria y secundaria. Sin embargo, los profesores con hijos mostraron menores niveles de burnout en los tres aspectos del síndrome.

De otro lado Byrne (1991, citado por Vandenberghe \& Huberman, 1999) en- 
contró que ambas variables no eran significativas para la misma población. En otro estudio los profesores de primaria y secundaria con hijos mostraron menores niveles de despersonalización (Pierce \& Mollow, 1990, citados por Vandenberghe \& Huberman, 1999). Se ha encontrado mayores niveles de realización personal en los profesores hombres, casados, de educación primaria en comparación con las profesoras y con los profesores(as) de secundaria. Asimismo, los sujetos casados muestran índices menores del síndrome del burnout que los no casados, no sólo en educadores sino también en trabajadores sociales, policías, entre otros (Gil \& Peiró, 1997). Sin embargo, cabe resaltar que en un gran porcentaje de estudios no se observa relaciones significativas entre ambas variables. Lo mismo sucede en relación con la presencia o ausencia de hijos pues no se observan resultados concluyentes ya que muy pocos estudios han tenido relaciones significativas entre la presencia o ausencia de hijos y el burnout.

Con respecto al grado de enseñanza, es más común entre los profesores de secundaria que en los profesores de primaria (Anderson \& Iwanicki, 1984; Beer \& Beer, 1992; Burke \& Greenglass, 1989; Feitler \& Tokar, 1982, Sckwab \& Iwanicki, 1982; Schwab et al., 1986, citados por Vandenberghe \& Huberman, 1999). En relación con los componentes del burnout, los profesores de secundaria presentan mayores niveles de despersonalización y de logros personales en comparación con los profesores de primaria (Anderson \& Iwanicki, 1984; Russel et al., 1987, citados por Vandenberghe \& Huberman, 1999).

En un estudio, Byrne (1991, citado por Vanderberghe \& Huberman, 1999) encontró que el tipo de estudiante tiene un efecto importante en el agotamiento emocional de los profesores de secundaria, así como en las percepciones de realización personal tanto en los profesores de secundaria como de universidad. Ha encontrado también que los profesores que enseñan a estudiantes graduados experimentan un mayor sentimiento de logro personal en comparación con los profesores que tienen estudiantes de pregrado.

Con respecto a los factores organizacionales los hallazgos han mostrado que el conflicto de roles es un factor determinante en la aparición del estrés laboral y en el burnout de los profesores (Benski et al., 1980; Capel, 1992; Jackson et al., 1986; Pettegrew \& Wolfe, 1982; Proctor \& Alexander, 1992, citados por Vandenberghe \& Huberman, 1999). Otros estudios muestran que el conflicto de roles está asociado con el agotamiento emocional (Burke \& Greenglass, 1995; Jackson et al., 1996; Pierce \& Molloy, 1990; Starnaman \& Miller, 1992; Schwab \& Iwanicki, 1982, citados por Vandenberghe \& Huberman, 1999) y con la despersonalización (Jackson et al., 1986; Schwab \& Iwa- 
nicki, 1982, citados por Vandenberghe \& Huberman, 1999). Sin embargo, Burke y Greenglass (1993) encontraron que el impacto del conflicto de rol en el burnout no es significativo.

Otros estudios señalan que el conflicto de roles presenta relaciones negativas significativas con los sentimientos de realización personal en el trabajo, y presenta relaciones significativas positivas con el agotamiento emocional y la despersonalización (Gil \& Peiró, 1997).

Con respecto a la ambigüedad de roles por la ausencia de claridad con relación a las obligaciones, derechos, objetivos, estatus y/o responsabilidad del trabajador se encuentran ciertos resultados contradictorios. Algunos autores piensan que la ambigüedad de roles es un predictor de la baja realización personal (Pierce \& Molloy, 1990; Schwab \& Iwanicki, 1982; Starnaman \& Miller, 1992 citados por Vandenberghe \& Huberman, 1999). Otros hallazgos señalan que la ambigüedad de roles es un predictor de agotamiento emocional (Burke \& Greenglass, 1995; Schwab \& Iwanicki, 1982, citados por Vandenberghe \& Huberman, 1999), mientras que otros muestran que es un predictor de la despersonalización (Pierce \& Molloy, 1990, citados por Vandenberghe \& Huberman, 1999). Friesen y Sarros (1989) encuentran que las predicciones con relación a la ambigüedad de roles, no son significativas.

En el caso de los educadores, la relación entre ambigüedad de rol y agota- miento emocional es más intensa que en los profesionales de enfermería (Gil, 1994). La relación entre la ambigüedad de rol y bajos sentimientos de realización personal es más intensa en el caso de los profesionales de enfermería que en los profesores (Gil, 1994).

El exceso de trabajo, que se refiere tanto a los componentes cuantitativos (muchas demandas y muy poco tiempo para realizarlas de manera adecuada) como cualitativos (complejidad del trabajo) es un desencadenante importante del síndrome de burnout, ya que supone un incremento de los sentimientos de agotamiento emocional de los profesionales (Gil \& Peiró, 1997).

Los reportes en profesores con respecto a esta variable como principal estresor son consistentes. Algunos factores son: exceso de trabajo de escritorio y de papeles, clases numerosas de estudiantes con habilidades heterogéneas, límites de tiempo y enseñar cursos que no coinciden con su área de especialización (Blase, 1986; Borg, 1990; Borg \& Riding, 1991; Borg et al., 1991; Byrne, 1991; Jenkins \& Calhoun, 1991; Proctor \& Alexander, 1992, citados por Vandenberghe \& Huberman, 1999). Los estudios encuentran que el exceso de trabajo contribuye al estrés del profesor y al burnout en general (Bensky et al., 1980; Borg \& Riding, 1991; Cooper \& Kelly, 1993; Jenkins \& Calhoun, 1991; O'Connor \& Clarke, 1990; Proctor \& Alexander, 1992, citados por Vandenberghe \& Huberman, 1999). 
Otros autores encuentran que está relacionado con el agotamiento emocional (Friesen et al., 1988; Fresen \& Sarros, 1989; Lutz \& Maddirala, 1990, citados por Vandenberghe \& Huberman, 1999).

En profesionales de enfermería se ha observado una relación significativa entre la sobrecarga laboral percibida y bajos sentimientos de realización personal en el trabajo (Richardsen et al., 1992; Gil et al., 1993; citados por Gil \& Peiró, 1997).

El ambiente del salón de clases influye también de manera crítica en las actitudes del profesor hacia la enseñanza, siendo éste un factor de estrés laboral. Los hallazgos sugieren que los problemas disciplinarios y el abuso el de los estudiantes, así como sus actitudes se asocian de manera significativa con el burnout del profesor (Bacharach et al., 1986; Borg et al., 1991, Punch \& Tuettemann, 1990, citados por Vandenberghe \& Huberman, 1999). La mala conducta de los alumnos así como sus actitudes negativas han sido consideradas como los principales estresores de los profesores (Borg et al., 1991; Okebukola \& Jegede, 1989; Hiebert, 1985 citados por Vandenberghe \& Huberman, 1999). Borg y Riding (1991, citados por Vandenberghe \& Huberman, 1999) encuentran que un clima inadecuado del salón como consecuencia del mal comportamiento de los estudiantes es más estresante para los profesores jóvenes que para los mayores.
Otro estresor importante para los profesores es la falta de participación en las decisiones que están relacionadas directamente con la calidad de vida en su trabajo. La poca participación en el proceso de toma de decisiones de la organización parece ser un factor importante en la disminución de la moral, satisfacción laboral, locus de control y autoestima de los profesores (Farber, 1991; McLaughlin et al., 1986, citado por Vandenberghe \& Huberman, 1999), siendo los principales efectos el estrés laboral y el burnout.

En el estudio del burnout del profesor se observan frecuentes referencias a la falta de apoyo por parte del personal administrativo. Sin embargo, los hallazgos son inconsistentes. Algunos autores encuentran una relación positiva entre el apoyo de los supervisores y el burnout del profesor (Sarros \& Sarros, 1992; Bacharach et al., 1986, citados por Vandenberghe \& Huberman, 1999), mientras que otros autores no encuentran una relación significativa (Burke \& Greenglass, 1993, citados por Vandenberghe \& Huberman, 1999). Existe cierto consenso en señalar que el apoyo de los compañeros de trabajo es muy importante en la disminución del estrés laboral (Maslach \& Jackson, 1994; Farber \& Miller, 1981, citados por Vandenberghe \& Huberman, 1999). Sin embargo, algunos estudios reportan que este apoyo no es efectivo (Cecil \& Forman, 1990). Otros estudios muestran que el soporte 
social en el trabajo presenta efectos directos significativos sobre las tres dimensiones del inventario Maslach de burnout (MBI) (Gil \& Peiró, 1997).

Se han encontrado también relaciones positivas significativas entre los sentimientos de agotamiento emocional y la falta de seguridad en el puesto de trabajo en los maestros (Anderson \& Iwanicki, 1984, citados por Gil \& Peiró, 1997).

Con respecto a los factores de personalidad, se ha encontrado que los profesores con un locus de control externo son más propensos a desarrollar el síndrome de burnout (Farber, 1991; Kyriacou, 1987; McLaughlin et al., 1986, Cedoline, 1982, citados por Vandenberghe \& Huberman, 1999). Se ha encontrado también un efecto significativo del locus de control externo en el agotamiento emocional (Lutz \& Maddirala, 1990; Mazur \& Lynch, 1989, citados por Vandenberghe \& Huberman, 1999); sin embargo, otros resultados muestran una asociación débil (Lunenburg y Cadavid, 1992, citados por Vandenberghe \& Huberman, 1999). Lunenburg y Cadavid (1992, citados por Vandenberghe \& Huberman, 1999) reportaron correlaciones de .25 entre el locus de control externo y la despersonalización y de -29 con la realización personal en el trabajo.

Diversos estudios sugieren que la $a u$ toestima está fuertemente asociada con el burnout (Farber, 1991; Hogan \& Hogan, 1982; Maslach, 1982; Motowidlo et al., 1986, citados por Vandenberghe
\& Huberman, 1999). Estudios anecdóticos (McLaughlin et al., 1986; Phillips y Lee, 1980, citados por Vandenberghe \& Huberman, 1999) han encontrado una fuerte evidencia de baja autoestima entre los profesores, como consecuencia de algunos factores antecedentes (por ejemplo, falta de apoyo del personal administrativo).

Otros investigadores reportaron que la autoestima es un predictor significativo del burnout (Friedman \& Farber, 1992). Se ha encontrado que los sujetos con más alta autoestima muestran bajos sentimientos de agotamiento emocional, bajas actitudes de despersonalización y altos sentimientos de realización personal en el trabajo (Gil \& Peiró, 1997).

Los estilos de afrontamiento planteados por Lazarus (Lazarus y Folkman, 1984) estarían relacionados con el estrés y el burnout. Los resultados de estas investigaciones señalan que las estrategias de afrontamiento activas en situaciones tensas ayudan a disminuir el estrés y el burnout. Estilos de afrontamiento poco activos (de escape, negación, resignación, menosprecio, etc.) parecen favorecer la aparición del estrés y del burnout (Rudow, citado por Vandenberghe \& Huberman, 1999). Los sujetos que emplean estrategias de carácter activo o centradas en el problema, muestran menos sentimientos de burnout que los sujetos que emplean estrategias de evitación/escape o centradas en la emoción (Ceslowitz, 1989; 
Boyle et al., 1991; Leiter, 1991, citados por Gil \& Peiró, 1997).

Con respecto a la personalidad tipo $A$, Mazur y Lynck (1989, citados por Vandenberghe \& Huberman, 1999) encuentran correlaciones positivas significativas con la despersonalización y la realización personal. En otros estudios se ha encontrado que el patrón conductual de tipo A permite diferenciar sujetos con altos y bajos sentimientos de burnout. Así, las personas tipo A se sentían significativamente más afectadas por el síndrome de burnout de acuerdo con el MBI (Nowack, 1986, citado por Gil \& Peiró, 1997). Otras investigaciones encuentran que la personalidad tipo A se relaciona significativamente con un mayor sentimiento de agotamiento emocional y mayor despersonalización y su relación con una baja realización personal en el trabajo no resultó significativa en una muestra de profesores (Nagy \& Davis, 1985, citado por Gil \& Peiró, 1997).

Con respecto a la empatía, Mazur y Lynch (1989, citados por Vandenberghe \& Huberman, 1999) señalan que ésta permite predecir el agotamiento emocional.

Williams (1989) reporta que existe una correlación significativa entre la empatía y el agotamiento emocional y la disminución de los logros personales.

Asimismo, encuentra que las profesoras muestran mayores niveles de empatía que los profesores.

\section{Población Y MUESTRA DE ESTUdio}

La población estuvo conformada por los docentes de educación primaria que enseñan a menores; de acuerdo con el informe estadístico del Ministerio de Educación del 2000, existen 26.900 profesores, los cuales están agrupados de acuerdo con el tipo de colegio en el que enseñan y a la USE a la que pertenecen (Ministerio de Educación, 2000).

La muestra del presente estudio fue de 264 profesores de nivel primaria; 170 son profesores de centros educativos estatales y 94 de centros educativos privados. De ellos, 213 (80,70\%) son profesores del sexo femenino y 51 del sexo masculino $(19,30 \%)$ (tablas $\mathrm{N}^{\circ} 1 \mathrm{y}$ $\mathrm{N}^{\mathrm{o}} 2$ ). Los profesores que participaron en la investigación tienen una dedicación de tiempo completo (40 horas) y un mínimo de cinco años trabajando como docentes.

\section{Inventario burnout de Maslach (MBI)}

El Inventario burnout de Maslach fue desarrollado por Maslach y Jackson en 1986 y se caracteriza por asumir que el desgaste psíquico es considerado como un síndrome caracterizado por sentimientos de agotamiento emocional, despersonalización y baja realización personal en el trabajo; es evaluado por el Maslach Burnout Inventory, que es un instrumento constituido por 22 ítems que se valoran con una escala tipo Likert de siete puntos. 
Tabla $\mathbf{N}^{\circ} 1$

Composición de la muestra por USE

\begin{tabular}{lccc}
\hline USE & $\begin{array}{c}\text { Profesores de } \\
\text { colegios estatales }\end{array}$ & $\begin{array}{c}\text { Profesores de } \\
\text { colegios particulares }\end{array}$ & Total profesores \\
\hline USE 1 & 14 & 6 & 20 \\
USE 2 & 29 & 13 & 42 \\
USE 3 & 27 & 14 & 41 \\
USE 4 & 14 & 15 & 28 \\
USE 5 & 21 & 10 & 31 \\
USE 6 & 18 & 10 & 28 \\
USE 7 & 16 & 7 & 23 \\
USE 8 & 13 & 13 & 26 \\
Callao & 18 & 7 & 25 \\
Total & 170 & 94 & 264 \\
\hline
\end{tabular}

Tabla No 2

Composición de la muestra por género y tipo de colegio

\begin{tabular}{|c|c|c|c|}
\hline \multirow[b]{2}{*}{ Tipo de colegio } & \multicolumn{2}{|c|}{ Género } & \multirow[b]{2}{*}{ Total } \\
\hline & Varón & Mujer & \\
\hline \multirow[t]{2}{*}{ Estatal } & 35 & 135 & 170 \\
\hline & $13,30 \%$ & $51,10 \%$ & $64,40 \%$ \\
\hline \multirow[t]{2}{*}{ Particular } & 16 & 78 & 94 \\
\hline & $6,10 \%$ & $29,5 \%$ & $35,60 \%$ \\
\hline \multirow[t]{2}{*}{ Total } & 51 & 213 & 264 \\
\hline & $19,30 \%$ & $80,70 \%$ & $100,00 \%$ \\
\hline
\end{tabular}

Los resultados de los análisis factoriales realizados en diferentes muestras con el MBI, arroja en la mayoría de los casos la existencia de tres factores ortogonales que son denominados agotamiento emocional, despersonalización y realización personal en el trabajo. Estos factores fueron obtenidos en estudios realizados con diferentes grupos de profesionales como maestros, enfermeros y personal sanitario, trabajadores sociales, farmacéuticos, entre otros
(Gold Roth, Wright \& Michael, 1991; Gil \& Peiró, 1997; Maslach, Jackson \& Leiter, 1997).

Para el presente estudio la versión de la prueba que se va a utilizar es el Educators Survey (ES) o MBI forma Ed, desarrollada por Maslach, Jackson y Schwab en 1986 (en Gold Roth, Wright \& Michael, 1991), que viene a ser una adaptación para maestros y profesores en la que la palabra "paciente" es sustituida por "alumno", permaneciendo 
igual el resto del cuestionario. Gil y Peiró (1997) reportan los estudios de Iwanicki y Schwab (1981) con una muestra de 469 maestros, y Gold (1984, en Gil \& Peiró, 1997) con otra muestra de 462 maestros que prueban la validez factorial de esta versión del MBI. Los valores alfa de Cronbach, del primer estudio, fueron: alfa $=0,90$ para agotamiento emocional, alfa $=0,76$ para despersonalización, y alfa $=0,72$ para realización personal en el trabajo. En el segundo estudio los valores fueron alfa $=0,88$, alfa $=0,74$ y alfa $=$ 0,72 , respectivamente.

Dado que la prueba tiene un origen inglés y una traducción española, se procedió a evaluar la equivalencia conceptual de los ítems y la equivalencia lingüística, utilizando para ello el criterio de jueces, con el fin de garantizar que el instrumento corresponda a nuestro contexto, tal como propone Marín (1986).

La validez del instrumento fue estudiada inicialmente a través de la validez de contenido por criterio de jueces. Para cuantificar los hallazgos se aplicó el coeficiente V de Aiken (Escurra 1988), los resultados presentados en la tabla $\mathrm{N}^{\mathrm{o}} 21$ (véase anexo) indican que todos los ítems alcanzan niveles adecuados de significación estadística, por lo que es posible trabajar con el instrumento sin realizar mayores modificaciones, pues presenta validez de contenido.

También se estudió la validez de constructo, por medio del método in- traprueba, efectuándose el Análisis Factorial Confirmatorio de manera que se trató de establecer si los tres factores obtenidos en la versión original de la prueba se mantenían en los datos de la muestra trabajada. Los resultados alcanzados en la tabla $\mathrm{N}^{\mathrm{o}} 3$ permiten observar que el modelo de tres factores alcanza mejores resultados que el modelo independiente, de manera que se observa un estadístico chi-cuadrado $(47,01)$ que no es significativo, a la vez que el análisis de residuales indica que los valores son inferiores al criterio de 0.05 , y también que los estadísticos encargados de evaluar la adecuación del modelo $(\mathrm{GFI}=0,98$ y AGFI $=0,95)$ son adecuados, por lo que se concluye que el modelo es corroborado en los datos evaluados y, por lo tanto, el Inventario de burnout de Maslach presenta validez de constructo.

El análisis del área de Agotamiento emocional del Inventario de Maslach, presentado en la tabla $\mathrm{N}^{\circ} 4$, nos permite apreciar que todos los ítems alcanzan correlaciones ítem-test corregidas superiores a 0,27 . En tanto que la confiabilidad estudiada por medio del coeficiente alfa de Cronbach ascendió a 0,78 , lo que nos lleva a concluir que los ítems de esta área permiten obtener puntajes confiables.

El análisis del área de despersonalización del Inventario de Maslach (véase tabla $\mathrm{N}^{\mathrm{o}} 5$ ), observamos que todos los ítems alcanzan correlaciones significativas entre 0,22 y 0,34 , por lo que todos 
Tabla $N^{\circ} 3$

Análisis factorial confirmatorio del Inventario burnout de Maslach

\begin{tabular}{lcc}
\hline Datos & Modelo de 3 factores & Modelo independiente \\
\hline Parámetros & 47 & 22 \\
Chi-cuadrado mínimo & 47,01 & 152,54 \\
G.L. & 206 & 231 \\
P &, 823 &, 020 \\
Chi-cuadrado mínimo/G.L. & 0,23 & 0,65 \\
RMR & 0,02 & 0,13 \\
GFI & 0,98 & 0,80 \\
AGFI & 0,95 & 0,86 \\
\hline
\end{tabular}

$N=334$

Tabla No 4

Análisis de ítems y confiabilidad del área de Agotamiento emocional del Inventario de Maslach

\begin{tabular}{cccc}
\hline Item & M & D.E. & ritc \\
\hline 1 & 2,31 & 1,76 & $0,57^{*}$ \\
2 & 3,13 & 1,87 & $0,52^{*}$ \\
3 & 1,87 & 1,83 & $0,57^{*}$ \\
6 & 1,13 & 1,67 & $0,49^{*}$ \\
8 & 2,27 & 1,88 & $0,59^{*}$ \\
13 & 0,73 & 1,32 & $0,43^{*}$ \\
14 & 1,27 & 1,72 & $0,38^{*}$ \\
16 & 1,30 & 1,77 & $0,44^{*}$ \\
20 & 1,46 & 1,03 & $0,27^{*}$ \\
\hline
\end{tabular}

Alfa de Cronbach $=0,78$ *

${ }^{*} p<.05 \quad \mathrm{~N}=334$

Tabla No 5

Análisis de ítems y confiabilidad del área de Despersonalización del Inventario de Maslach

\begin{tabular}{cccc}
\hline Item & $M$ & D.E. & $r_{\text {itc }}$ \\
\hline 5 & 1,16 & 1,59 & $0,22^{*}$ \\
10 & 1,13 & 1,04 & $0,23^{*}$ \\
11 & 0,77 & 1,46 & $0,27^{*}$ \\
15 & 0,69 & 1,65 & $0,33^{*}$ \\
22 & 0,60 & 1,15 & $0,34^{*}$ \\
\hline
\end{tabular}

Alfa de Cronbach $=0.76$ *

${ }^{*} \mathrm{p}<.05 \quad \mathrm{~N}=334$ 
pueden ser retenidos. La confiabilidad evaluada a través del coeficiente alfa de Cronbach es de 0,76. Estos resultados nos permiten concluir que los ítems de esta área logran obtener puntajes confiables.

En lo que concierne al área de Realización personal en el trabajo del Inventario de Maslach, presentada en la tabla $\mathrm{N}^{\mathrm{o}} 6$, se encuentra que los ítems alcanzan correlaciones ítem-test corregidas significativas entre 0,27 y 0,46 ; también se observa que el coeficiente alfa de Cronbach es de 0,74 , lo cual signifi- ca que los ítems del área permiten obtener puntajes confiables.

Los resultados han permitido corroborar la validez y confiabilidad del Inventario burnout de Maslach (MBI), por lo que es factible utilizar el instrumento en la investigación, pero, debido a que no existe consenso respecto de cuándo se presenta el agotamiento emocional (burnout), se ha considerado pertinente analizar las puntuaciones de cada una de las áreas que conforman el instrumento.

Tabla $N^{\circ} 6$

Análisis de ítems y confiabilidad del área de Realización personal del Inventario de Maslach

\begin{tabular}{cccc}
\hline İtem & $M$ & D.E. & $r_{\text {itc }}$ \\
\hline 4 & 0,92 & 1,63 & $0,42^{*}$ \\
7 & 1,38 & 1,94 & $0,43^{*}$ \\
9 & 1,09 & 1,93 & $0,43^{*}$ \\
12 & 1,02 & 1,74 & $0,27^{*}$ \\
17 & 1,25 & 1,89 & $0,39{ }^{*}$ \\
18 & 0,90 & 1,64 & $0,42^{*}$ \\
19 & 0,96 & 1,69 & 0,46 * \\
21 & 1,56 & 1,92 & $0,39 *$ \\
\hline
\end{tabular}

Alfa de Cronbach $=0,74$ *

${ }^{*} p<.05 \quad \mathrm{~N}=334$

Tabla $N^{0} 7$

Bondad de ajuste a la curva normal de Kolmogorov-Smirnov de las variables estudiadas

\begin{tabular}{lcccc}
\hline Variable & Media & D.E. & K-S Z & P a dos colas \\
\hline Agotamiento emocional & 15,58 & 9,46 & 1,67 &, 0730 \\
Despersonalización & 4,38 & 4,48 & 2,67 &, $0127^{*}$ \\
Realización personal & 12,49 & 6,49 & 2,03 &, $0215^{*}$
\end{tabular}

* $\mathrm{p}<.05$

$\mathrm{N}=264$ 


\section{RESUltados}

El análisis de la bondad de ajuste a la curva normal da como resultado que el área de agotamiento emocional presenta coeficientes K-S Z que alcanzan niveles de probabilidad muy elevados, lo que indicaría que sus puntajes tienen una aceptable aproximación a la distribución normal. Sin embargo, en el caso de la despersonalización y la realiza- para el análisis de los datos consideramos factible la utilización de estadísticos paramétricos con el fin de obtener resultados más precisos.

\section{Análisis según variables de comparación}

Pasemos ahora a describir el análisis comparativo según las variables sociodemográficas de nuestro estudio.

Tabla $\mathbf{N}^{\circ} 8$

Prueba $Z$ de comparación de medias de los puntajes de las variables estudiadas por género

\begin{tabular}{lrrrrr}
\hline Variables & \multicolumn{2}{c}{$\begin{array}{c}\text { Varón } \\
\text { N = 51 }\end{array}$} & \multicolumn{2}{c}{ Mujer } & N $=213$ \\
& M & DE & M & DE & \\
\hline Agotamiento emocional & 11,96 & 6,76 & 16,44 & 9,82 & $-3,09$ *** \\
Despersonalización & 5,27 & 4,84 & 4,16 & 4,37 & 1,59 \\
Realización personal & 11,86 & 6,57 & 12,64 & 6,48 & $-0,77$ \\
\hline
\end{tabular}

${ }^{\star \star \star} p<, 001$

ción personal los resultados indican que existen diferencias estadísticas significativas, por lo cual en esas variables no existe una adecuada semejanza a la curva normal. Tal como indican Hair et al. (1999), el que una variable no presente una distribución normal no determina necesariamente el uso de estadísticas no-paramétricas, dado que algunas estadísticas paramétricas (como el ANOVA de uno y de dos factores, la prueba $\mathrm{Z}$ de comparación de medias) son lo suficientemente robustas como para permitir su empleo con variables cuyos datos no se aproximen a la distribución normal. Es por ello que
El análisis comparativo de las variables estudiadas de acuerdo al género, presentado en la tabla $\mathrm{N}^{\circ} 8$, permite apreciar que sólo existen diferencias significativas en el caso del agotamiento emocional $(Z=-3,09, p<, 05)$, en el que las mujeres alcanzan puntuaciones más elevadas $(\mathrm{M}=16,44 \mathrm{DE}=9,82)$ que los varones $(\mathrm{M}=11,96, \mathrm{DE}=6,76)$.

La tabla $\mathrm{N}^{\circ} 9$ resume los resultados del análisis comparativo de acuerdo con el tipo de gestión educativa.

La comparación de acuerdo con el tipo de gestión educativa, (véase tabla $\mathrm{N}^{\mathrm{o}}$ 9), permite apreciar la presencia de diferencias estadísticas significativas 
Tabla $N^{\circ} 9$

Prueba $Z$ de comparación de medias de los puntajes de las variables estudiadas por tipo de gestión educativa

\begin{tabular}{|c|c|c|c|c|c|}
\hline \multirow[t]{2}{*}{ Variables } & \multicolumn{2}{|c|}{$\begin{array}{l}\text { Estatal } \\
\mathrm{N}=170\end{array}$} & \multicolumn{2}{|c|}{$\begin{array}{c}\text { Particular } \\
\mathrm{N}=94\end{array}$} & \multirow[t]{2}{*}{ Z } \\
\hline & M & DE & M & $\mathrm{DE}$ & \\
\hline Agotamiento emocional & 15,80 & 9,51 & 15,17 & 9,40 & 0,52 \\
\hline Despersonalización & 4,63 & 4,68 & 3,92 & 4,08 & 1,22 \\
\hline Realización personal & 13,29 & 6,81 & 11,03 & 5,62 & $2,74^{* *}$ \\
\hline
\end{tabular}

${ }^{* *} p<, 01$

en la realización personal $(\mathrm{Z}=2,74, \mathrm{p}<$ ,05). Los profesores que laboran en colegios estatales alcanzan, como puede observarse, valores más elevados en la realización personal que los que trabajan en colegios particulares.

Veamos seguidamente, las comparaciones de acuerdo con el lugar de procedencia.

En lo que concierne al análisis por lugar de procedencia, en la tabla $\mathrm{N}^{\circ} 10$ apreciamos que existen diferencias estadísticas significativas en despersonalización $(Z=-1,99, p<, 05)$ y la reali- zación personal $(\mathrm{Z}=2,48, \mathrm{p}<, 05)$. En la realización personal los profesores que proceden de Lima ( $M=13,55$ $\mathrm{DE}=7,09)$ alcanzan puntuaciones más elevadas que los de provincias $(\mathrm{M}=11$, $59 \mathrm{DE}=5,81$ ), mientras que en despersonalización los profesores de provin$\operatorname{cias}(\mathrm{M}=4,88 \mathrm{DE}=4,59)$ obtienen mayores puntajes que los de Lima $(\mathrm{M}=$ 3,78 $\mathrm{DE}=4,36)$.

Seguidamente, se ofrecen los resultados del análisis comparativo de acuerdo con el hecho de si los profesores trabajan en otra institución o no lo hacen.

Tabla $N^{\circ} 10$

Prueba $Z$ de comparación de medias de los puntajes de las variables estudiadas por lugar de procedencia

\begin{tabular}{lrrrrr}
\hline Variables & \multicolumn{2}{c}{ Lima } & \multicolumn{2}{c}{ Provincia } & $\mathrm{Z}$ \\
& $\mathrm{M}$ & $\mathrm{N}=121$ & \multicolumn{2}{c}{$\mathrm{N}=143$} & \\
& 16,21 & 9,80 & 15,04 & 9,17 & 1,01 \\
\hline Agotamiento emocional & 3,78 & 4,36 & 4,88 & 4,59 & $-1,99$ * \\
Despersonalización & 13,55 & 7,09 & 11,59 & 5,81 & 2,48 * \\
Realización personal & & & &
\end{tabular}

* $\mathrm{p}<, 05$ 
Tabla No 11

Prueba $Z$ de comparación de medias de los puntajes de las variables estudiadas por trabaja en otra institución

\begin{tabular}{lrrrrr}
\hline Variables & \multicolumn{2}{c}{ Sí trabaja } & \multicolumn{2}{c}{ No trabaja } & Z \\
& \multicolumn{1}{c}{$\mathrm{N}=56$} & $\mathrm{DE}$ & $\mathrm{M}$ & $\mathrm{DE}$ & \\
\hline Agotamiento emocional & 17,82 & 11,46 & 14,97 & 8,78 & 2,01 * \\
Despersonalización & 5,02 & 4,58 & 4,21 & 4,44 & 1,20 \\
Realización personal & 14,46 & 7,40 & 11,96 & 6,14 & 2,59 * \\
\hline
\end{tabular}

* $\mathrm{p}<, 05$

La comparación, de acuerdo con la pregunta de si trabaja en otra institución (véase tabla $\mathrm{N}^{0} 11$ ), indica que existen diferencias estadísticas significativas en agotamiento emocional $(\mathrm{Z}=$ 2,01, $\mathrm{p}<, 05)$, y realización personal $(\mathrm{Z}=2,59, \mathrm{p}<, 05)$, apreciándose que los profesores que trabajan en otra institución alcanzan valores más elevados en agotamiento emocional y realización personal.

La tabla que se presenta a continuación contiene el análisis comparativo por estado civil de los profesores.
El análisis comparativo de acuerdo con el estado civil permite observar que sólo existen diferencias significativas en el caso del agotamiento emocional $(Z=2,45, p<, 05)$, en el que los solteros obtienen puntuaciones más elevadas $(M=17,70 \mathrm{DE}=9,26)$ que los casados $(\mathrm{M}=14,62, \mathrm{DE}=9,51)$.

A continuación se dan a conocer los resultados del análisis de varianza de un factor por centro de formación académica de los profesores (véase tabla $\mathrm{N}^{\mathrm{o}} 13$ ).

En lo que respecta a la comparación del centro de formación académica de

Tabla $N^{\circ} 12$

Prueba Z de comparación de medias de los puntajes de las variables estudiadas por estado civil

\begin{tabular}{lrrrrr}
\hline Variables & \multicolumn{2}{c}{ Soltero(a) } & \multicolumn{2}{c}{ Casado(a) } & Z \\
& \multicolumn{2}{c}{$\mathrm{N}=88$} & \multicolumn{1}{c}{$\mathrm{N}=152$} & \\
& $\mathrm{M}$ & $\mathrm{DE}$ & $\mathrm{M}$ & $\mathrm{DE}$ & \\
\hline Agotamiento emocional & 17,70 & 9,26 & 14,62 & 9,51 & 2,45 * \\
Despersonalización & 4,74 & 8,08 & 4,26 & 4,22 & 0,79 \\
Realización personal & 12,51 & 6,77 & 12,47 & 6,41 & 0,05 \\
\hline
\end{tabular}

* $p<, 05$ 
los docentes, no se encuentran diferencias estadísticas significativas en ninguna de las variables estudiadas.

Seguidamente presentamos la tabla $\mathrm{N}^{\mathrm{o}} 14$, en la cual se exponen los resultados del análisis de varianza de un factor por grupos etarios.

En las comparaciones por grupo de edad, no se encuentran diferencias significativas (véase tabla $\mathrm{N}^{\circ} 14$ ).

Respecto de las comparaciones por tiempo de servicio no existen diferencias estadísticas significativas (tabla $\mathrm{N}^{\circ}$ 15).
La tabla $\mathrm{N}^{\circ} 16$ da a conocer los resultados del análisis de varianza de un factor por número de hijos; no arroja diferencias estadísticas significativas en caso alguno.

A renglón seguido se presenta el análisis de varianza de dos factores del nivel de realización personal según el centro de formación académica y el tipo de gestión educativa.

El análisis de varianza de dos factores de la realización personal según el tipo de centro de formación académica y el

Tabla No 13

Análisis de Varianza de un factor por centro de formación académica

\begin{tabular}{lcccc}
\hline Variables & U. nacional & U. privada & Inst. superior & \\
& $\mathrm{N}=105$ & $\mathrm{~N}=77$ & $\mathrm{~N}=82$ & $\mathrm{~F}$ \\
& $\mathrm{M}$ & $\mathrm{DE}$ & $\mathrm{M}$ & $\mathrm{DE}$ \\
\hline Agotamiento emocional & 15,82 & 15,23 & 15,59 & 0,08 \\
Despersonalización & 4,21 & 3,82 & 5,12 & 1,82 \\
Realización personal & 12,23 & 12,86 & 12,48 & 0,21 \\
\hline
\end{tabular}

* $\mathrm{p}<, 05 \quad \mathrm{GL}_{1}=2 \quad \mathrm{GL}_{2}=261$

Comparaciones significativas con el test de Scheffé $=$ a

Tabla No 14

Análisis de varianza de un factor por grupo de edad

\begin{tabular}{lccccc}
\hline Variables & $\begin{array}{c}27 \text { a } 30 \text { años } \\
\mathrm{N}=55\end{array}$ & $\begin{array}{c}31 \text { a } 40 \text { años } \\
\mathrm{N}=118\end{array}$ & $\begin{array}{c}41 \text { a } 50 \text { años } \\
\mathrm{N}=63\end{array}$ & $\begin{array}{c}51 \text { a más } \\
\mathrm{N}=28\end{array}$ & $\mathrm{~F}$ \\
& $\mathrm{M}$ & $\mathrm{M}$ & $\mathrm{M}$ & $\mathrm{M}$ & \\
\hline Agotamiento emocional & 16,10 & 15,74 & 15,97 & 12,96 & 0,82 \\
Despersonalización & 5,15 & 4,58 & 3,43 & 4,14 & 1,60 \\
Realización personal & 12,73 & 12,84 & 11,95 & 11,75 & 0,40 \\
\hline
\end{tabular}

* $\mathrm{p}<, 05 \mathrm{GL}_{1}=3 \quad \mathrm{GL}_{2}=260$

Comparaciones significativas con el test de Scheffé $=a$ 
Tabla No 15

Análisis de varianza de un factor por tiempo de servicio

\begin{tabular}{lccccc}
\hline Variables & 5 a 10 años & 11 a 15 años & 16 a 20 años & 21 a más & $\mathrm{F}$ \\
& $\mathrm{N}=95$ & $\mathrm{~N}=80$ & $\mathrm{~N}=48$ & $\mathrm{~N}=41$ & \\
& $\mathrm{M}$ & $\mathrm{M}$ & $\mathrm{M}$ & $\mathrm{M}$ & \\
\hline Agotamiento emocional & 15,58 & 15,51 & 17,73 & 13,17 & 1,73 \\
Despersonalización & 4,65 & 4,21 & 4,83 & 3,54 & 0,80 \\
Realización personal & 11,86 & 13,68 & 13,00 & 11,02 & 2,00
\end{tabular}

$\mathrm{GL}_{1}=3 \quad \mathrm{G} \cdot \mathrm{L}_{2}=260$

Comparaciones significativas con el test de Scheffé $=a$

tipo de gestión educativa del colegio permite observar que sólo existen diferencias estadísticas significativas en el caso de la gestión educativa $(\mathrm{F}=7,17$, $\mathrm{p}<, 01$ ), no observándose la presencia de interacciones entre las variables analizadas.

Tabla $\mathbf{N}^{\circ} 16$

Análisis de varianza de un factor por número de hijos

\begin{tabular}{lcccc}
\hline Variables & Ninguno & 1 a 3 & 4 a más & $\mathrm{F}$ \\
& $\mathrm{N}=93$ & $\mathrm{~N}=151$ & $\mathrm{~N}=20$ & \\
& $\mathrm{M}$ & $\mathrm{M}$ & $\mathrm{M}$ & \\
\hline Agotamiento emocional & 17,01 & 15,00 & 13,20 & 1,99 \\
Despersonalización & 4,61 & 4,25 & 4,30 & 0,19 \\
Realización personal & 12,40 & 12,69 & 11,40 & 0,36
\end{tabular}

$\mathrm{GL}_{1}=2 \mathrm{GL}_{2}=261$

Tabla № 17

Análisis de varianza de dos factores del nivel de realización personal según centro de formación académica y gestión educativa

\begin{tabular}{lcccc}
\hline Gestión & \multicolumn{3}{c}{ Centro de formación académica } & Total \\
& U. estatal & U. particular & Inst. superior & \\
\hline Estatal & 13,01 & 13,43 & 13,57 & 13,29 \\
& $\mathrm{~N}=72$ & $\mathrm{~N}=47$ & $\mathrm{~N}=51$ & $\mathrm{~N}=170$ \\
Particular & 10,52 & 11,97 & 10,68 & 11,03 \\
& $\mathrm{~N}=33$ & $\mathrm{~N}=30$ & $\mathrm{~N}=31$ & $\mathrm{~N}=94$ \\
Total & 12,23 & 12,86 & $12,48=82$ & 12,49 \\
& $\mathrm{~N}=105$ & $\mathrm{~N}=77$ & $\mathrm{~N}=82$ & $\mathrm{~N}=264$ \\
\hline
\end{tabular}

$\mathrm{F}$ del centro de formación académica $=0,34$

$\mathrm{F}$ del tipo de gestión educativa $=7,17$ **

$\mathrm{F}$ de interacción $=0,248$

** $\mathrm{p}<, 01\left[\mathrm{GL}_{1}=2 \mathrm{GL}_{2}=1 \mathrm{GL}_{3}=260\right]$ 
Clasificación del desgaste psíquico (burnout) basada en los baremos de los docentes evaluados

A continuación presentamos la tabla correspondiente a un análisis cualitativo de cada una de las dimensiones del desgaste psíquico (burnout).
En lo que concierne al nivel de realización personal, se observa que los profesores exhiben en el $50 \%$ niveles altos, en tanto que el $28,8 \%$ alcanza niveles medios, y el $21,2 \%$ niveles bajos.

Tabla No 18

Nivel de agotamiento emocional, despersonalización y realización personal de los docentes de primaria

\begin{tabular}{lccccrr}
\hline \multirow{2}{*}{ Nivel } & \multicolumn{2}{c}{ Agotamiento emocional } & \multicolumn{2}{c}{ Despersonalización } & \multicolumn{2}{c}{ Realización personal } \\
& Frecuencia & $\%$ & Frecuencia & $\%$ & Frecuencia & $\%$ \\
\hline Alto & 89 & 33,7 & 87 & 33,0 & 132 & 50,0 \\
Medio & 90 & 34,1 & 82 & 31,0 & 76 & 28,8 \\
Bajo & 85 & 32,2 & 95 & 36,0 & 56 & 21,2 \\
Total & 264 & 100,0 & 264 & 100,0 & 264 & 100,0 \\
\hline
\end{tabular}

Como se puede apreciar en la tabla $\mathrm{N}^{\circ}$ 18 , existe un $33,7 \%$ de los participantes que presentan altos niveles de agotamiento emocional y un $34,1 \%$ con niveles medios, vale decir que más del $67 \%$ de la muestra tendría un indicador elevado para la presencia del desgaste psíquico.

En el caso del nivel de despersonalización que presentan los docentes, encontramos que el 33\% de los participantes alcanzan niveles altos y el $31 \%$ niveles medios, lo cual nos indica que más del $65 \%$ de los profesores evaluados presentan el segundo indicador elevado para la presencia del desgaste psíquico.

\section{Clasificación del desgaste psíquico} (burnout) basada en el modelo de Golembiewski

En esta sección se ha optado por trabajar con el modelo de Golembiewski (1983). En primer lugar presentaremos las fases del desgaste psíquico y después los niveles respectivos.

En la tabla $\mathrm{N}^{\circ} 19$ se observa que los porcentajes más elevados se ubican en la fase III $(18,2 \%)$ y en la fase VI (20,8\%). Las fases V, VII y VIII agrupan un total de $33,4 \%$ de los casos, mientras que las fases II y IV el 9,5\%, y la fase I el más bajo porcentaje de frecuencias $(8,7 \%)$. Los resultados arrojan diferencias significativas en el 
Tabla $\mathbf{N}^{\circ} 19$

Fases del desgaste psíquico específico de los docentes de primaria

\begin{tabular}{lccl}
\hline Fase & Frecuencia & Porcentaje & Nivel \\
\hline $\mathrm{I}$ & 23 & 8,7 & \\
$\mathrm{II}$ & 25 & 9,5 & Bajo \\
III & 48 & 18,2 & \\
\hline $\mathrm{IV}$ & 25 & 9,5 & Medio \\
$\mathrm{V}$ & 29 & 11,0 & \\
\hline $\mathrm{VI}$ & 55 & 20,8 & Alto \\
VII & 29 & 11,0 & \\
VIII & 30 & 11,4 & \\
\hline Total & 264 & 100,0 &
\end{tabular}

$X^{2}=29,64 \quad$ G.L. $=7 \quad p=, 0001$

porcentaje de casos que corresponden a cada fase $\left(X^{2}=29,64 \quad\right.$ G.L. $=7$ $\mathrm{p}=, 0001)$.

La reclasificación de los resultados de la tabla anterior en tres niveles, presentados en la tabla $\mathrm{N}^{\circ} 20$, indica que el $43,2 \%$ de los casos alcanzan niveles altos, en tanto que el $36,3 \%$ niveles bajos y el $20,5 \%$ valores medios. Estas diferencias son significativas $\left(X^{2}=21,55\right.$ G.L. $=2 p=, 0000)$.

Tabla $N^{\circ} 20$ Nivel de desgaste psíquico general de los docentes de primaria

\begin{tabular}{lcc}
\hline Nivel & Frecuencia & Porcentaje \\
\hline Alto & 114 & 43,2 \\
Medio & 54 & 20,5 \\
Bajo & 96 & 36,3 \\
Total & 264 & 100,0 \\
\hline
\end{tabular}

$X^{2}=21,55 \quad$ G.L. $=2 p=, 0000$
Estos hallazgos indican que los docentes en más del 63,8\% estarían expuestos a desarrollar a corto plazo el síndrome de desgaste psíquico.

Resulta evidente que en sociedades como la peruana la profesión del docente está sometida a los avatares y dificultades que, en general, caracterizan la vida de sus habitantes. Por esto, las carencias y limitaciones que afectan a los peruanos en general, también alcanzan a los docentes. Históricamente, aunque todos los regímenes han proclamado su preocupación por la educación y por mejorar las condiciones del trabajo docente (Contreras y Cueto, 2000), en la práctica la educación, la remuneración de los docentes y la inversión educativa han sido mínimas y tratadas como no prioritarias. Esto ha llevado a que desde muchos años atrás se hable de que la educación peruana está en crisis, por lo cual se requiere una reformulación de ella a fondo. 
El fenómeno del desgaste psíquico (burnout), ha sido estudiado y continúa siéndolo precisamente en el marco del desempeño de las llamadas profesiones sociales a las que hemos hecho referencia (Berg, 1994; Sarros \& Sarros, 1992). Profesores que trabajan con niños cuyas dificultades de aprendizaje son de gran envergadura, enfermeras que se desempeñan en salas de cuidados de pacientes terminales, pueden ser mencionados como ejemplo de profesionales en quienes puede presentarse el burnout.

El agotamiento emocional y despersonalización son dos de las características definitorias del fenómeno del desgaste psíquico. El agotamiento emocional supone cansancio y fatiga, tanto en el plano físico como en el psíquico. Por su parte, la despersonalización significa sentimientos, actitudes y respuestas negativas, distantes y frías hacia otras personas. Los profesores de la muestra investigada trabajan en condiciones subjetivas de mínima motivación y experimentan su trabajo como una actividad cargada de desafíos de múltiples tensiones, de constantes estímulos negativos, que ponen a prueba y al final afectan su capacidad de adaptación y su rendimiento.

Situaciones extremas de trabajo ejercen, pues, la sensación subjetiva de estrés experimentada por el docente, así como lo que podríamos llamar una sensación de extrañeza y alejamiento del trabajo que es la despersonalización.
En los resultados por género se encuentran diferencias entre hombres y mujeres, en la dimensión agotamiento emocional. Para este hallazgo es posible formular varias interpretaciones. En primer lugar, podemos remitirnos al estereotipo fuertemente difundido de que la mujer tiene menos resistencia física que el hombre y que, por lo tanto, sus umbrales de agotamiento son más bajos, mientras que los hombres, a los que por lo general se atribuye mayor vigor y resistencia física, así como una más sólida estabilidad emocional, presentarían menos indicadores de agotamiento emocional. Estos estereotipos, que provienen de la evolución histórica de las nociones del sexo masculino y femenino, conservan hasta hoy vigencia, como lo demuestran investigaciones recientes (León \& Sirlopú, 1996).

Como se ha señalado, esto corresponde a un estereotipo y, por tanto, debe ser tomado con cuidado como elemento explicativo. Debe reconocerse, sin embargo, que algo de ese estereotipo debería ser analizado. El ejercicio de una profesión supone, obviamente, un mayor nivel de educación (Bárrig, 1988), y exige, además, conductas de mucha afirmación; es decir, "conductas más masculinas", lo que puede demandar un mayor esfuerzo emocional. Y, como ya se señaló en líneas atrás, la profesión docente va asociada a elevados niveles de estrés.

Mayor plausibilidad ofrece la alternativa que plantea que la mujer, por lo 
general, aparte del trabajo remunerado, cumple en casa trabajos que son considerados inherentes a la condición femenina y que se pueden resumir bajo el rubro de actividades hogareñas. La literatura permite afirmar que las mujeres, después del trabajo, al retornar al hogar, se enfrentan con una jornada laboral no menos intensa, que incluye la preparación de los alimentos, la atención de los niños y del esposo, así como la supervisión o realización de las tareas domésticas relacionadas con el orden y el aseo, todo lo cual no suele ser considerado como trabajo cuando en realidad sí lo es (Guzmán \& Portocarrero, 1985). Y que además va asociado a niveles de estrés que no son adecuadamente percibidos, pues subsiste aún la idea de que el hogar es un lugar seguro y de descanso (Ragúz de Romaña, 1998). Esta segunda alternativa nos parece la más pertinente si se tiene en cuenta que en todas las demás variables consideradas en el análisis no se registran diferencias significativas entre hombres y mujeres, lo cual va en contraposición del estereotipo que presenta a las mujeres como más débiles, más proclives a desórdenes emocionales $\mathrm{y}$ menos resistentes al estrés.

Pasemos ahora a la variable gestión educativa. Como se sabe, en el Perú existen escuelas privadas y públicas. Esta división va asociada a diferencias marcadas en una serie de aspectos, desde la infraestructura física hasta las remuneraciones. En la percepción del público en general, y muy en especial en la de los padres de familia, la educación que se imparte en las instituciones privadas es la de mejor calidad y la que, por tanto, garantiza mayores niveles de competitividad para sus hijos (Ansión et al., 1998). El examen de estos hallazgos nos permite verificar que los profesores que trabajan para entidades estatales presentan valores más elevados en realización personal que los que se desempeñan en entidades particulares.

Esto puede ser explicado probablemente por el hecho de que muchos profesores que trabajan para el Estado provienen de universidades nacionales y la condición de profesor supone, para ellos un significativo paso en su movilidad social vertical, mientras que los profesores que se desempeñan en entidades particulares provienen sobre todo del estrato social medio y perciben, por tanto, con más claridad las limitaciones en el prestigio social que tiene la profesión docente.

Sin embargo, cabe anotar que la variable realización personal es, por su naturaleza misma, de gran complejidad, por lo cual su interpretación exige particular cautela, más aún en el caso de nuestro trabajo, en donde los resultados en realización personal no siguen la distribución de la curva normal.

Considerando la variable lugar de procedencia, ésta resulta de importancia en el presente estudio, porque en el Perú, país de marcada centralización, la calidad de vida y el proceso mismo de la existencia de las personas suelen ser 
muy diferentes en la capital que en el resto del país. El estudio efectuado por López (1997) ha puesto de relieve cuán grande es la distancia que hay en la vida de las personas, según transcurra en la costa, en la sierra, en la selva o en la capital.

Como se sabe, hoy un elevado porcentaje de la población de Lima está conformado por personas que han llevado a cabo un proceso de migración interna (Balbi, 1997). Esto también se puede aplicar a los integrantes de la muestra con la cual se ha investigado. Además, se observa que, en lo referido al lugar de procedencia, existen diferencias significativas en despersonalización y en realización personal. En el caso de la despersonalización es posible reconocer que los profesores de provincia obtienen puntajes más elevados, lo que indicaría que ellos experimentan mayores sentimientos de extrañeza y de distanciamiento con respecto a lo que realizan y a ellos mismos. Estos sentimientos suelen ser considerados frecuentes por parte de quienes emigran, pues resultan del desarraigo que acompaña el abandono del lugar de origen para trasladarse al nuevo lugar de residencia.

En lo que se refiere a realización personal, los limeños obtienen promedios más elevados que los provincianos. Es probable que en sus puntajes haya jugado un papel de importancia el hecho de que ellos se desenvuelven en un medio geográfico y social que les es fami- liar desde muy temprano, razón por la cual los esfuerzos de adaptación a éste han sido mínimos, en contraste con lo sucedido con los profesores que se han trasladado a la capital desde las diferentes provincias.

En lo que concierne a la variable trabaja o no trabaja en otra institución, se encontraron diferencias significativas en agotamiento emocional y realización personal. Estas diferencias eran de esperar: los docentes de la muestra que trabajan en varias instituciones obtuvieron puntajes más elevados en agotamiento emocional y en realización personal, lo cual resulta comprensible si se tiene en cuenta que el pluriempleo (una realidad laboral de alta frecuencia en la docencia en el Perú) supone una mayor cantidad de horas de trabajo y el consiguiente cansancio, así como la duplicación de los esfuerzos laborales.

Teniendo en consideración que la labor docente supone un prolongado trabajo de preparación previa de la clase, así como de dictado de ésta, es posible explicar que los niveles de agotamiento emocional sean mayores en aquellos docentes que se desempeñan en varias instituciones. De otro lado, sin embargo, los promedios más elevados en realización personal se pueden entender a la luz de los mayores ingresos y tal vez la mayor sensación de la propia utilidad y competitividad que probablemente están vinculados al pluriempleo. Es necesario señalar en este aspecto, en apoyo a esta suposición, que el trabajo, 
la actividad laboral que uno desempeña, es central en la identidad psicosocial así como en la estabilidad emocional (Roazen, 1997).

En lo que respecta a la variable estado civil, se encuentra que los solteros obtuvieron puntuaciones más elevadas que los casados en agotamiento emocional. Este hallazgo puede entenderse a la luz del hecho de que redes sociales de mayor amplitud, así como la presencia de una familia nuclear en torno al individuo sirven de mayor y más adecuado soporte emocional, y que la soledad, física o subjetiva, da lugar a mayor estrés y a una valoración más negativa de la propia situación. Esta suposición puede confirmarse a través del hecho de que varios estudios demuestran que personas que puntúan más alto en introversión son más proclives a experimentar burnout que los extrovertidos (Davis-Johnson, 1991; Lemkau et al., 1988). Como realidad psicosocial, la soledad ha sido estudiada en fecha aún reciente en nuestro medio (Zambrano Mora, 1997), encontrándose que es percibida como un sentimiento negativo, poco promocionador de la salud mental y, por tanto, debe evitarse.

El análisis de varianza de dos factores del nivel de realización personal, según el centro de formación académica y gestión educativa, permite afirmar que sólo existen diferencias estadísticas significativas en el caso de la gestión educativa. Es interesante remarcar, sin embargo, que quienes obtienen el punta- je más alto de realización personal en la gestión educativa estatal son los docentes que provienen de institutos superiores, mientras que en la gestión educativa particular los que puntúan más alto son precisamente los que provienen de universidades particulares. Este hecho puede llevarnos a plantear que aquellos profesores que provienen de institutos superiores, que, por lo general, son personas de una extracción social muy modesta, perciben su trabajo en entidades estatales como un ascenso social, lo cual explicaría el porqué de su más elevado puntaje en realización personal.

Pasemos ahora a considerar los resultados del análisis del desgaste psíquico (burnout) tomando en cuenta el modelo de las fases de Golembiewski (Golembiewski et al., 1983).

Este autor propone ocho fases progresivas del desarrollo del desgaste psíquico, e identifica el nivel IV como el crítico, por considerar que es en este nivel en el cual la persona comienza a experimentar los efectos de dicho desgaste, cuya posterior evolución tiende a incrementarse en los niveles superiores.

Los resultados permiten afirmar que el $43,2 \%$ de la muestra presenta niveles elevados (fases VI, VII, VIII). Esto reflejaría la fuerte influencia negativa proveniente de las condiciones particularmente duras y estresantes en que los profesores tienen que desenvolverse en el Perú. En refuerzo a lo señalado debe anotarse que un $20 \%$ se encuentra en las fases IV y V. 
Es el factor agotamiento emocional el que ejerce una influencia positiva en la despersonalización. El término agotamiento emocional es uno de los de más larga tradición en la psicología. Cansancio mental, neurastenia, surmenage, son algunos de los sinónimos empleados a lo largo de la historia de la psicología para designar un estado subjetivo y muchas veces también objetivo de cansancio físico, de saturación afectiva, de progresiva pérdida de sentido de trabajo, $\mathrm{y}$ de una creciente insatisfacción con el medio circundante (Shorter, 1997).

Por otro lado, la despersonalización provocada por el agotamiento emocional proviene de la pérdida de significado de trabajo que experimenta la persona. Ya hemos dicho que el trabajo es un elemento central, nuclear del desarrollo de la identidad personal. Erikson (Friedman, 1999), el gran teórico de la identidad, ha destacado el inmenso significado que tiene el trabajo para el hombre en el mundo de hoy.

El trabajo no sólo responde a necesidades económicas, sino que también tiene implicancias emocionales, de estatus, y, por supuesto, de realización personal. Sin embargo, trabajos rutinarios, mal remunerados, con escaso prestigio, o que se cumplen en condiciones de extrema dificultad, no contribuyen al desarrollo de una identidad sólida, sino, por el contrario, la cuestionan o la debilitan.

Ese parece ser el caso de los profesores con los que se ha trabajado. En lo referente al agotamiento emocional, encontramos que $67 \%$ de la muestra presenta un indicador elevado de éste. No menos significativo es el hecho de que el $65 \%$ de los docentes presente niveles altos o medios de despersonalización. Asimismo, que el 31\% de los encuestados se ubique en el nivel bajo de realización personal, y el $28 \%$ en el medio, corrobora la imagen poco favorable del grupo de encuestados, en lo que concierne a aquello que podríamos llamar su situación existencial.

La moderna psicología organizacional, la psicología de la personalidad y la psicología de la salud son tres áreas aplicadas de la psicología que parten del presupuesto de que el ser humano sólo puede realizarse y llevar a cabo una vida con sentido, allí donde las condiciones ambientales son favorables y estimuladoras para su desarrollo personal. Situaciones de permanente frustración, de constante desafío a la capacidad de adaptación de los individuos y en las que, además, las carencias y limitaciones son el denominador común, tienden a causar desmoralización, ausencia de interés en las tareas que se tienen que realizar, reacciones afectivas negativas y a configurar, finalmente, un estado psicológico al que Seligman dio el nombre de desesperanza aprendida (Seligman, 1975).

Más allá de la discusión que se ha llevado a cabo, la conclusión más importante es que el gremio de profesores es un grupo sometido a particulares fuer- 
zas estresoras, con muy escasas posibilidades de reconocimiento social, y, lamentablemente, con una motivación laboral puesta a prueba por las circunstancias particularmente adversas en las que tiene que trabajar. Cualquier intento de mejorar la educación en el Perú debe pasar por una reforma sustantiva de las condiciones objetivas, infraestructurales y humanas en las que los profesores cumplen con su delicada tarea día tras día. 


\section{REFERENCIAS}

Adams, E. (1999). Vocational teacher stress and internal characteristics. Journal of Vocational and Technical Education, 16, (1), Fall.

Alarcón, R. (1991). Métodos y diseños de investigación del comportamiento. Lima: Universidad Peruana Cayetano Heredia, Fondo Editorial.

Ansión, I.; Lazarte, A.; Matos, S.; Rodríguez, J. \& Vega-Centeno, P. (1998). Educación: la mejor herencia. Lima: Pontificia Universidad Católica del Perú.

Arbuckle, J. (1999). AMOS 4.0: Programming reference guide. Chicago: Small Waters Corporation.

Babbie, E. (2000). Fundamentos de la investigación social. México: International Thomson Editores.

Balbi, C.R. (Ed.) (1997). Lima: Aspiraciones, reconocimiento y ciudadanía en los noventa. Lima: Pontificia Universidad Católica del Perú.

Banco Mundial (1996). Prioridades para la educación: Examen del Banco Mundial. Washington DC.

Bandura, A. (1989). Human agency in social cognitive theory. American Psychologist, 44, (9), 1175-1184.

Bárrig, M. (1998). Empleo y trabajo femenino. Revista Peruana de Ciencias Sociales, 1, 7-39.

Berg, B.D. (1994). Educator burnout revisited: voices from the staff room. The Clearing House, 67 (4), 185-188.

Bibou-Nakou, I.; Stogiannidou, A. \& Kiosseoglou, G. (1999). The relation between teachers burnout and teachers' attributions and practices regarding school behaviour problems. School Psychology International, 20 (2), 209217.

Blase, J.J. (1986). A quantitative analysis of sources of teacher stress: consequences for performance. American Educational Research Journal, 23, 13-39.

Boles, J.; Dean, D.H.; Ricks, J.M.; Short, J.C. \& Wang (2000). The dimensionality of the Maslach Burnout Inventory across small business owners and educators. Journal of Vocational Behavior, 56 (1): 12-34.

Brannon, L. \& Feist, J. (1992). An introduction to behavior and health. Belmont, California: Wadsworth.

Brouwers, A. \& Tomic, W. (2000). A longitudinal study of teacher burnout and perceived self-efficacy in classroom management. Teaching and Teacher Education, 16 (2), 239-253.

Brown, F. (1986). Principios de la medición en psicología y educación. México: El Manual Moderno.

Bruner, J. (1995). Desarrollo cognitivo y educación. Madrid: Ediciones Morata.

Burke, R.J. \& Greenglass, E.R. (1989a). Correlates of psychological burnout phase among teachers. Journal of Health and Human Resources Administration, 12, 46-62.

Burke, R.J. \& Greenglass, E.R. (1993). Work stress, role conflict, social support and psychological burnout among teachers. Psychological Reports, 73, 371-380.

Byrne, B.M. (1989). A primer of LISREL: Basic applications and programming for Confirmatory Analytic Models. New York: Springer-Verlag Inc. 
Byrne, B.M. (1998). Structural equation modeling with LISREL, PRELIS, and SIMPLIS: Basic concepts, applications and programming. New Jersey: Lawrence Erlbaum Associates Publishers.

Cecil, M.A. \& Forman, S.G. (1990). Effects of stress inoculation training and coworker support groups on teacher's stress. Journal of School Psychology, 28, 105-118.

Cherniss, C. (1980a). Professional burnout in human service organization. New York: Praeger.

Cherniss, C. (1980b). Staff burnout: Job stress in the human service. Beverly Hill: Sage.

Cherniss, C. (1992). Long-term consequences of burnout: An exploratory study. Journal of Organizational Behavior, 13 (1), 1-11.

Contreras, C. \& Cueto M. (2000). Historia del Perú contemporáneo. Lima. Pontificia Universidad Católica del Perú/Instituto de Estudios Peruanos.

Davis-Johnson, L.B. (1991). The effect of psychological type in stress coping resources and professional bournout. Dissertation Abstracts International, 52, (03), 1765B.

De las Cuevas, C.; De la Fuente, J.A.; Aliviana, M. \& Ruíz-Benitez, A. (1995). Desgaste profesional y clima laboral en atención primaria. Fundación Mapfre Medicina, 6, (1). www. mapfremedicina.es/publicaciones/espa nyol/ v6n1art1.htm

Delors, J. (1996). La educación encierra un tesoro. Lima: Unesco.

Díaz, M. \& Saavedra, J. (2000). La carrera del maestro en el Perú: Factores ins- titucionales, incentivos económicos y desempeño. Lima: Grade, Documento de Trabajo 32, 1-64.

Dunham, J. (1984). A framework of teacher's coping strategies for a whok school stress management policy. Educational Management and Administration, 22, 168-174.

Dunham, J. \& Varma, V. (Eds.) (1998). Stress in teachers. Past, present, and future. Londres: Whurr Publishers Ltd.

Edelwich, J. \& Brodsky, A. (1980). Burnout: Stages of disillisuonment in the helping professions. New York: Human Sciences Press.

Escurra, L.M. (1988). Cuantificación de la validez de contenido por el criterio de jueces. Revista de Psicología, 6 (1 y 2), 103-111.

Farber, B.A. (1983). Stress and burnout in the human service professions. New York: Pergamon, General Psychology Press.

Farber, B.A. (1984). Stress and burnout in suburban teachers. Journal of Educational Research, 77, (6), 325-331.

Farber, B.A. (1991). Crisis in education. Stress and burnout in the american teacher. San Francisco: Jossey-Bass Publisers.

Farber, B.A. (1999). Inconsequentiality, the key to understanding teacher burnout. $A$ sourcebook of international research and practice. New York, Cambridge University Press, $X V$, 362, 159-165.

Feldman, R. (1998). Psicología con aplicaciones a los países de habla hispana. Bogotá: McGraw-Hill/ Interamericana Editores.

Fiadzo, E., Golembiewski, T., Luo, H., 
Bradbury, M. \& Rivera, T. (1996). Burnout in ghanaian hospitals: phase model findings in sub-saharan Africa. Journal of Public Administration and $\mathrm{Ma}$ nagement: An interactive journal, 1. num. 1. http://www.hbg.psu.edu/ faculty/ jxr11/golem1.html.

Freudenberger, H.J. (1974). Staff burnout. Journal of Social Issues, 30, 159-165.

Freudenberger, H.J. (1975). The staff burnout syndrome in alternative institutions. Psychotherapy: Theory, Research \& Practice, 12, 72-83.

Friedman, I.A. \& Farber, B. (1992). Professional self-concept as a predictor of teacher burnout. Journal of Educational Research, 86, 28-35.

Friedman, L.J. (1999). Identity's architect. A biography of Erik H. Erikson. New York: Scribner.

Friesen, D. \& Sarros, J.C. (1989). Sources of burnout among educators. Journal of Organizational Behavior, 10, 179-188.

Fuller, N. (1993). Dilemas de la femineidad. Lima: Pontificia Universidad $\mathrm{Ca}-$ tólica del Perú.

Fuller, N. (1995). Identidades masculinas. Lima: Pontificia Universidad Católica del Perú.

Gil, P. (1994). "El síndrome de burnout: Un modelo multicausal de antecedentes y consecuentes en profesionales de enfermería". Tesis para optar el grado de doctor. Universidad de la Laguna, Facultad de Psicología.

Gil, P. \& Peiró, J. (1995). A causal model of burnout process development: An alternative to Golembiewski and Leiter models. Seven European Congress on
Work and Organizational Psychology, Györ (Hungary).

Gil, P. \& Peiró, J. (1997). Desgaste psíquico en el trabajo: El síndrome de quemarse. Madrid: Editorial Síntesis.

Gold, Y.; Roth, R.A.; Wright, C.R. \& Michael, W.B. (1991). The relationship of scores on the educators survey, A modified version of the Maslach burnout inventory, to three reaching-related variables form a sample of 132 beginning teachers. Educational and Psychological Measurement, 51, 429-438.

Golembiewski, R.T.; Munzenrider, R. \& Carter, D. (1983). Phases of progressive burnout and their work site covariants: Critical issues in OD research and praxis. Journal of Applied Behavioral Science, 19 (4), 461-481.

Goodwin, C.J. (1998). Research in Psychology; Methods and design. New York: John Wiley \& Sons, In.

Graziano, A.M. \& Raulin, M.L. (1989). Research methods, a process of inquiry. Albany: Harper Collins Publishers.

Griffith, J.; Steptoe, A.; Cropley, M. (1999). An investigation of coping strategies associated with job stress in teachers. British Journal of Educational Psychology. 69 (4): 517-531.

Grimm, L.G. \& Yarnold, P.R. (1995). Reading and understanding Multivariate statistics. Washington DC: American Psychological Association.

Grimm, L.G. \& Yarnold, P.R. (2000). Reading and understanding more multivariate statistics. Washington DC: American Psychological Association.

Guzmán, V. \& Portocarrero, A. (1985). Dos veces mujer. Lima: Mosca Azul. 
Hair, J.F., Anderson, R.E., Tatham, R.L. \& Black, W.C. (1999). Análisis multivariante. México: Prentice Hall International.

Hernández, S.R., Fernández, C.C. \& Baptista, L.P. (1998). Metodología de la Investigación. México: McGraw-Hill.

Hock, R.R. (1998). Professional burnout among public school teachers. Public Personal Management, 17, 167-189.

Ivancevich, J.M. \& Matteson, M.T. (1992). Estrés y trabajo. México: Editorial Trillas.

Iwanicki, E.F. \& Schwap, R.C. (1981). A cross validation study of the Maslach Burnout Inventory. Educational and Psychological Measurement, 41, 4, 1167-1174.

Jackson, S.E. \& Maslach, C. (1982). Burnout: A concept in need of refinement. Paper presented at the Meeting of the American Psychological Association, Washington.

Jöreskog, K. \& Sörbom, D. (1998). LISREL 8: Structural equation modeling with the SIMPLIS command language. New Jersey: Lawrence Erlbaum Associates Publishers.

Kyriacon, C. (1980). Stress, health and school-teachers: A comparison with other professions. Cambridge: Journal of Education, 10, 154-159.

Kyriacon, C. (1985). Teacher stress and burnout: An interactional review. Educational Research, 29, 2, 146-152.

Lazarus, E. \& Folkman, S. (1984). Stress, appraisal and coping. New York: Springer.

Lemkau, J.P.; Purdy, R.P.; Rafferty, J.P. \& Rudisill, J.R. (1988). Correlates of bur- nout among family practice residents. Journal of Medical Education, 63, 9, 682-691.

León, R. \& Sirlopú, D. (1996). “Diferencias de género en el patrón de conducta tipo A en un grupo de estudiantes universitarios de Lima metropolitana". Revista de Psicología, 14, 183-209.

Lohr, S. (1999). Muestreo: Diseño y análisis. México: International Thomson Editores.

López, S. (1997). Ciudadanos reales e imaginarios. Concepciones, desarrollo y mapas de la ciudadanían en el Perú. Lima: Instituto de Diálogo y Propuesta.

Llor, B.; Abad. M.A.; García, M. \& Nieto, J. (1998). Ciencias psicosociales aplicadas a la salud. Madrid: Interamérica/ McGraw-Hill.

Maccoby, E.E. (1998). Gender as a social category. Developmental Psychology, 24, 755-765.

Marín, G. (1986). Consideraciones metodológicas básicas para conducir investigaciones en América Latina. Acta Psiquiátrica y Psicológica de América Latina, 32, 183-192.

Maslach, C. (1982). The cost of caring. Englewood Cliffs, NJ: Prentice Hall.

Maslach, C. \& Jackson, S.E. (1981). The measurement of experienced burnout. Journal of Ocupational Behavior, 12, 99-113.

Maslach C. \& Jackson, S.E. (1986). MBI: Maslach Burnout Inventory. Manual Research Edition. Palo Alto: University of California, Consulting Psychologists Press.

Maslach, C.; Jackson, S.E. \& Leiter, M.P. (1997). Maslach Burnout Inventory: 
Third Edition. Chapter in C.P. Zalaquett \& R.J. Wood (Eds.), Evaluating stress: $A$ book of resources. Lanham, MD: Scarecrow Press.

Mc Keachie, W. J. (1986). Teaching tips. A guidebook for the beginning college teacher. 8a. edition. Lexington DC.: Heath and Company.

Ministerio de Educación (1995). Censo Escolar 1993. Lima: MED.

Mueller, R.O. (1998). Basic principles of structural equation modeling: An introduction to LISREL and EQS. New York: Springer-Verlag Inc.

Peiró, J. (1987). Psicología de la organización. Madrid: Universidad Nacional de Educación a Distancia.

Pérez, C. (2000). Técnicas de muestreo estadístico: Teoría, práctica y aplicaciones informáticas. México: Alfaomega Grupo Editor, S.A. de C.V.

Ragúz de Romaña, M. (1998). La mujer que trabaja: Revisión de investigaciones sobre atribución de logro. Preferencias y metas ocupacionales, actitud hacia la mujer que trabaja. Revista de Psicología, 6, 87-101.

Ramos, F. (1999) El síndrome de burnout. Madrid: UNED- FUE.

Reid, J. (1999). The relationships among personality type, coping strategies, and burnout in elementary teachers. Journal of Psychological Type, 51, 22-33.

Rice, J. (1997). Desarrollo humano. México: Mundo: Prentice-Hall.

Roazen, P. (1997). Erik H. Ericson, the power and limits of a vision. Northvale, (New Jersey), London: Jason Aronson.

Rosenholtz, S. (1989). Teachers workplace. White Plains, NY: Longman.
Salgado, A.; Yela, J.R.; Quevedo, M.P.; Delgado, N.; Fuentes, J.M.; Sánchez, A.; Sánchez, T. \& Velasco, C. (1997). El síndrome de "burnout": Estudio empírico en profesores de enseñanza primaria. Iber Psicología, 18, 1-17.

Salkind, N.J. (2000). Métodos de investigación. México: Prentice-Hall/Hispanoamericana S.A.

Sánchez C.H. \& Reyes C. (1998). Metodología y diseños en la investigación cientifica. Lima: Editorial Mantaro.

Sandín, B. (1999) El estrés psicosocial. Conceptos y consecuencias clínicas. Madrid: UNED-FUE.

Sara-Lafosse, V.; Cordano, D. \& Gentges, I. (1994). ¿Formando maestros discriminadores? Lima: Pontificia Universidad Católica del Perú.

Sarros, J.C. \& Sarros, A.M. (1992). Social support and teacher burnout. Journal of Educational Administration, 37, 55-69.

Schwab, R. (1986). Burnout in education. In C. Maslach y S.E. Jackson. Maslach Burnout Inventory: Manual. Palo Alto: Consulting Psychologist Press.

Seligman, M.E.P. (1975). Helplessness: On depression development and death. San Francisco: W.H. Freeman.

Shorter, E. (1997). A history of psychiatry. New York: John Wiley.

Sierra Bravo R. (1994a). Análisis estadístico multivariable. Teoría y ejercicios. Madrid: Editorial Paraninfo.

Sierra Bravo, R. (1994b). Tesis doctorales y trabajos de investigación científica. Madrid: Editorial Paraninfo.

Sierra Bravo, R. (1995). Técnicas de investigación social. Madrid: Editorial Paraninfo. 
Steiner, G. (1995). Después de Babel. México DF: Fondo de Cultura Económica.

Tarea (2000). Educación primaria al final de la década: Politicas curriculares en el Perú y los países andinos. Lima: Tarea.

Tatar, M., Yahav, V. (1999). Secondary school pupils' perceptions of burnout among teachers. British Journal of Educational Psychology, 69 (4): 457468.

Thompson, M.; Page, S. \& Coopers (1993). A test of Caver and Scheier's self-control model of stress in exploring burnout among mental health nurses. Stress Medicine, 9, 221-235.

Topf, M. (1989). Personality hardiness, occupational stress and burnout in critical case nurses. Research in Nursing and Health, 12, 179-186.

Travers, Ch. \& Cooper, C. (1997). El estrés de los profesores: La presión en la actividad docente. Barcelona: Paidós.

Tukey, J.W. (1977). Exploratory data analysis. Reading: Addison-Wesley.

Vandenberghe, R. \& Huberman, A.M. (1999). Understanding and preventing teacher burnout: A sourcebook of international research and practice. Cambridge: Cambridge University Press.

Van-Horn, J.E.; Schaufeli, W. \& Enzmann, D. (1999). Teacher burnout and lack of reciprocity. Journal of Applied Social Psychology, 29, 1, 91-108.

Visauta Vinacua B. (1986). Técnicas de investigación social. Modelos causales. Barcelona: Editorial Hispano Europea.

Williams, C.A. (1989). Empathy and burnout in male and female helping profes- sionals. Research in Nursing and Health, 12, 3, 169-178.

Zambrano Mora, A. (1997). Uno entre los demás. Lima: La Parola Editorial. 
Fernández.

Anexo

Tabla $N^{\circ} 21$

Validez de contenido del Inventario burnout de Maslach

\begin{tabular}{|c|c|}
\hline Ítem & V Aiken \\
\hline 1 & 1,00 * \\
\hline 2 & 1,00 * \\
\hline 3 & 1,00 * \\
\hline 4 & 0,89 * \\
\hline 5 & 0,89 * \\
\hline 6 & 0,89 * \\
\hline 7 & 0,89 * \\
\hline 8 & 0,89 * \\
\hline 9 & 0,89 * \\
\hline 10 & 0,89 * \\
\hline 11 & 0,89 * \\
\hline 12 & 0,89 * \\
\hline 13 & 0,89 * \\
\hline 14 & 0,89 * \\
\hline 15 & 0,89 * \\
\hline 16 & 1,00 * \\
\hline 17 & 0,89 * \\
\hline 18 & 0,89 * \\
\hline 19 & 0,89 * \\
\hline 20 & 0,89 * \\
\hline 21 & 0,89 * \\
\hline 22 & 1,00 * \\
\hline
\end{tabular}

${ }^{*} \mathrm{p}<, 05 \quad$ Jueces $=9$

Tabla $\mathbf{N}^{\circ} 22$

Baremo del Inventario burnout de Maslach (MBI)

\begin{tabular}{ccccc}
\hline Rango percentil & Agotamiento emocional & Despersonalización & Realización personal & Rango percentil \\
\hline 1 & 0 & & & 1 \\
5 & 2 & & 0 & 5 \\
10 & 5 & & 1 & 10 \\
15 & 6 & 0 & 2 & 20 \\
20 & 8 & & 3 & 25 \\
25 & 9 & & 4 & 30 \\
30 & 10 & 1 & 5 & 35 \\
35 & 11 & 2 & & 40 \\
40 & 12 & 3 & 6 & 45 \\
45 & 13 & & 7 & 50 \\
50 & 14 & 4 & 8 & 55 \\
55 & 15 & 5 & 9 & 60 \\
60 & 16 & 6 & 11 & 65 \\
65 & 17 & 7 & 12 & 70 \\
70 & 19 & 8 & 13 & 75 \\
75 & 20 & 9 & 14 & 80 \\
80 & 22 & 10 & 18 & 85 \\
85 & 27 & 11 & 20 & 90 \\
90 & 30 & 13 & 24 & 95 \\
95 & 36 & 20 & 44 & 99 \\
99 & 42 & 4,34 & 9,07 & D \\
M & 15,46 & 4,44 & 5,32 & \\
\hline E. & 63 & & & \\
\end{tabular}

$\mathrm{N}=334$ 


\section{Encuesta de docentes}

Este trabajo trata de indagar acerca de las características personales y sociales que presentan en la actualidad los profesores de nivel primaria de Lima metropolitana. Este estudio, cuyos resultados serán difundidos entre los maestros de Lima, nos permitirá conocer, de manera científica, la realidad de los docentes y realizar las recomendaciones correspondientes.

En ese sentido, solicitamos su colaboración para responder a los diferentes componentes de esta encuesta que es completamente anónima.

Muchas gracias

\section{Datos personales}

1. Edad
2. Sexo
(1) Masculino
(2) Femenino

3. Lugar de nacimiento:
(1) Lima
(2) Provincia
Especifique

4. Tipo de colegio donde trabaja:
(1) Nacional
(2) Privado laico
(3) Privado religioso

5. Actualmente enseña en:
(1) Primaria
Grado
(2) Secundaria
Año

6. ¿Realiza actividad docente en otra institución?
(1) Sí
(2) No

7. Años trabajando en docencia:

8. Formación académica:
(1) Universidad nacional
(2) Universidad privada
(3) Instituto
(4) Otros:

9. Especialidad

10. Estado civil:
(1) Soltero (a)
(4) Divorciado(a)
(2) Casado (a)
(5) Separado(a)
(3) Viudo (a)
(6) Conviviente

11. Número de hijos 


\section{Fernández,}

\section{Inventario de Maslach}

A continuación se presenta un conjunto de enunciados sobre diferentes ideas, pensamientos y situaciones relacionadas con su trabajo, usted debe indicar la frecuencia con que se presentan. Debe responder marcando con un aspa $(\mathrm{X})$ sobre el número que le corresponda, según la siguiente escala:

$\begin{array}{ll}\text { Nunca } & 0 \\ \text { Alguna vez al año o menos } & 1 \\ \text { Una vez al mes o menos } & 2 \\ \text { Algunas veces al mes } & 3 \\ \text { Una vez por semana } & 4 \\ \text { Algunas veces por semana } & 5 \\ \text { Todos los días } & 6\end{array}$

1. Debido a mi trabajo me siento emocionalmente agotado

2. Al final de la jornada me siento agotado

3. Me encuentro cansado cuando me levanto por las mañanas y tengo que enfrentarme a otro día de trabajo

4. Puedo entender con facilidad lo que piensan mis alumnos

$\begin{array}{lllllll}0 & 1 & 2 & 3 & 4 & 5 & 6\end{array}$

5. Creo que trato a algunos alumnos con indiferencia

6. Trabajar con alumnos todos los días es una tensión para mí

7. Me enfrento muy bien con los problemas que me presentan mis alumnos

8. Me siento agotado por el trabajo

9. Siento que mediante mi trabajo estoy influyendo positivamente en la vida de otros

10. Creo que me comporto de manera más insensible con la gente desde que hago este trabajo

11. Me preocupa que este trabajo me esté endureciendo emocionalmente

12. Me encuentro con mucha vitalidad

13. Me siento frustrado por mi trabajo

14. Siento que estoy haciendo un trabajo demasiado duro

15. Realmente no me importa lo que les ocurrirá a algunos de los alumnos a los que tengo a mi cargo en el colegio

16. Trabajar en contacto directo con los alumnos me produce bastante estrés

17. Tengo facilidad para crear una atmósfera relajada a mis alumnos

18. Me encuentro animado después de trabajar junto con mis alumnos

19. He realizado muchas cosas que valen la pena en este trabajo

20. En el trabajo siento que he llegado al límite de mis posibilidades

21. Siento que sé tratar de forma adecuada los conflictos emocionales en el trabajo

22. Siento que los alumnos me culpan de algunos de sus problemas 